\title{
Sensory wellbeing workshops for inpatient and day-care patients with anorexia nervosa
}

\author{
Kate Tchanturia (D) · C. Baillie · C. Biggs · A. Carr · A. Harrison · Z. Li · C. McFie $\cdot$ O. Oyeleye $\cdot$ C. Toloza
}

Received: 11 February 2021 / Accepted: 23 April 2021 / Published online: 15 June 2021

(C) Springer-Verlag GmbH Austria, ein Teil von Springer Nature 2021

\begin{abstract}
Summary
Background The wellbeing of patients with eating disorders is one of the priorities in the "bigger picture" of treatment for eating disorders. Sensory soothing strategies for sensory sensitivities are supportive tools which could be useful in day-care and inpatient clinical programmes.

Methods Evaluation of multiple separate sensory wellbeing workshops consisting of psychoeducation and experiential components delivered in inpatient and intensive day-care services was performed. Participants' self-report questionnaires were evaluated preand post-workshop. Additionally, patients' comments and qualitative feedback was collected after completion of the workshop.

Results There was strong evidence that self-reported awareness of sensory wellbeing, awareness of strategies to enhance sensory wellbeing, and confidence in managing sensory wellbeing increased after the workshops with positive qualitative feedback from participants. The feedback questionnaires highlighted that patients found the sessions useful and were able to
\end{abstract}

\section{Dr. K. Tchanturia $(\bowtie) \cdot \mathrm{Z}$. Li}

Department of Psychological Medicine, Institute of Psychiatry, Psychology and Neuroscience, King's College London, De Crespigny Park, PO59, London, SE5 8AF, UK kate.tchanturia@kcl.ac.uk

Dr. K. Tchanturia · C. Baillie · C. Biggs · A. Carr · A. Harrison · C. McFie $\cdot$ O. Oyeleye $\cdot$ C. Toloza

Eating Disorders National Service, South London and Maudsley NHS Foundation Trust, London, UK

Dr. K. Tchanturia

Ilia State University, Tbilisi, Georgia

A. Harrison

Department of Psychology and Human Development, University College London, Institute of Education, 25 Woburn Square, London, WC1H 0AA, UK use some of the skills and strategies they learned in the workshop.

Conclusion This pilot work on sensory wellbeing workshops with a protocol-based format was feasible and beneficial for the patient group. Preliminary evidence suggests that delivery of similar workshops could be sensible in addition to treatment as usual in inpatient and day-care programmes.

Keywords Eating disorders - Autistic spectrum condition - Anorexia nervosa - Sensory sensitivity · Wellbeing

Workshops zum sensorischen Wohlbefinden für stationäre und tagesklinische Patienten mit Anorexia nervosa

\section{Zusammenfassung}

Grundlagen Das Wohlbefinden von Patienten mit Essstörungen stellt einen der Schwerpunkte in der Behandlung von Essstörungen dar. Sensorische Beruhigungsstrategien sind unterstützende Maßnahmen, die für ambulante und stationäre klinische Programme von Nutzen sein könnten.

Methodik Untersucht wurden mehrere einzelne Workshops zum sensorischen Wohlbefinden, die aus Psychoedukation und der Arbeit mit den Erfahrungen bestanden und für stationäre sowie für ambulante Patienten abgehalten wurden. Die Fragebögen der Teilnehmer wurden vor und nach dem Workshop ausgewertet. Darüber hinaus wurden Kommentare und qualitative Rückmeldungen der Patienten nach Abschluss des Workshops gesammelt.

Ergebnisse Es gab deutliche Hinweise darauf, dass das subjektive Bewusstsein für sensorisches Wohlbefinden, das Bewusstsein für Strategien zur Verstärkung des sensorischen Wohlbefindens und das Vertrauen darin, sein sensorisches Wohlbefinden zu steuern, 
nach den Workshops zunahm, und es gab positive qualitative Rückmeldungen von den Teilnehmern. Anhand der Fragebögen zum Feedback stellte sich heraus, dass die Patienten die Workshops nützlich fanden und einige der Kompetenzen und Strategien anwendeten, die sie in dem Workshop gelernt hatten. Schlussfolgerung Dieses Pilotprojekt mit Workshops zum sensorischen Wohlbefinden mit einem protokollbasierten Format war für diese Patientengruppe geeignet und nützlich. Diese vorläufige Evidenz zeigt, dass ähnliche Workshops in Ergänzung zur üblichen Behandlung im stationären und ambulanten Rahmen zweckmäßig sein könnten.

Schlüsselwörter Essstörungen · Autismus-SpektrumStörungen · Anorexia nervosa $\cdot$ Sensorische Empfindlichkeit · Wohlbefinden

\section{Introduction}

Anorexia nervosa (AN) is a life-threatening psychiatric disorder associated with high levels of functional and social difficulties $[1,2]$. Recently, the focus has started to shift from treating only eating disorder (ED) symptoms to the "bigger picture" of recovery. Research exploring creative and novel ways to support patients with adjunct research-based treatments is rising [3].

In the context of wellbeing and recovery, the sensory system and good adjustment to the environment plays a critical role. Research into sensory sensitivities has started to produce interesting results. For example, research demonstrates that people with EDs avoid new sensory experiences. Kinnaird and colleagues demonstrated that patients with EDs, with and without autism spectrum comorbidity, are hypersensitive to smells, touch and noise, and hypersensitivity in different domains could be addressed in their treatment [4-6]. Clinical audit data shows that almost $37 \%$ of people with AN treated in inpatient and day-care programmes have high Autism Spectrum Condition (ASC) traits and are therefore likely to experience some sensory differences [7].

In the novel clinical pathway for autism and eating disorders, PEACE (Pathway for Eating disorders and Autism developed from Clinical Experience), we have started to measure sensory sensitivities in patients and have developed psychoeducation materials and experiential activities to support sensory wellbeing [8]. We also found that patients with no autistic traits as well as high autistic traits benefited from the sensory changes made in the dining room evidenced with focus groups [9]. Based on the research evidence $[4,10]$, we have developed one-off workshops which offer both psychoeducational content and experiential activities to support patients by creating a soothing and helpful sensory toolkit.

The key purposes of this small pilot are to: (a) examine the feasibility of the sensory wellbeing workshops in ED inpatient and day-care treatment pro- grammes, (b) to evaluate feedback from study participants, and (c) discuss possible future developments and how to generate further evidence-based sensory workshops.

\section{Methodology}

\section{Participants}

All patients who participated in the study were female adults (aged 18-60 years) who had a DSM-5 [11] diagnosis of AN (binge-purge, restrictive subtype) and were part of the intensive (inpatient or day care) programmes in the South London and Maudsley NHS Foundation Trust (SLaM) National Eating Disorder Service. Patients with a diagnosis of bulimia nervosa, binge-eating disorder, or other eating disorder diagnosis were excluded from analysis, in order to focus on AN during this pilot phase. Ethical approval was granted by the local research and governance committee at SLaM.

Demographic information used in this study was provided by patients at the start of their admission to the treating service.

\section{Self-report measures}

Autism spectrum quotient score, short version (AQ-10) The AQ-10 is included in a battery of clinical measures which patients are invited to complete at the start of their admission to the treating service. The AQ-10 is a 10 item questionnaire devised from the Autism Spectrum Quotient. It is designed to enable screening for presence of ASC traits. A score greater than 6 is indicative of potential autistic spectrum condition [12].

Pre-workshop (T1) and post-workshop (T2) sensory wellbeing questionnaire

All workshop participants were given a questionnaire to complete at the start and end of the workshop (Appendix Fig. 2). The questionnaire used a 5-point Likert scale ranging from 1 ("Not aware/confident at all") to 5 ("Really aware/confident") to enable participants to rate: how aware they are of their sensory wellbeing, how aware they are of strategies to enhance their sensory wellbeing, and how confident they feel to manage their own sensory wellbeing.

\section{Post-workshop feedback questionnaire}

In addition to the post-workshop sensory wellbeing questionnaire, all workshop participants were given a feedback form at the end of the session with two open-ended questions asking what they liked most about the sessions and if they had any other comments. 


\section{Procedure}

Each of the workshops were made available to all patients receiving treatment in the relevant service (inpatient or day care). In each case patients were made aware of the workshops in community meetings and through posters and sign-up sheets. At the start and end of the workshop participants were asked to complete the pre (T1)/post (T2) workshop sensory wellbeing questionnaires, described above. The workshops ran for between one and one a half hours and were each facilitated by two staff from the service psychological therapies team.

\section{Description of the intervention}

The intervention was a one-off sensory workshop, aiming to increase awareness about the sensory system, explore how the sensory system can help with self-regulation, identify strategies that enhance sensory wellbeing and provide participants with the language and tools to communicate their sensory needs. The workshop included psychoedcuation and facilitated discussion between participants about their sensory experiences, followed by two exercises. One exercise allowed participants to explore a variety of sensory materials in order to identify their sensory preferences. The second exercise was a Do It Yourself (DIY) activity in which participants made their own sensory item, for example a scented hand cream. Further psychoeducation and tools to identify and communicate sensory preferences were also provided.

In light of the COVID-19 (coronavirus disease 2019) lockdown, the workshop was also adapted to run online so that patients in the clinical services that were running virtually were able to participate. Online versions of the pre- and post-workshop feedback questionnaires and sensory booklet were distributed and an online flyer was circulated before the workshop encouraging participants to bring along items suggested for the sensory toolkit.

The online sensory wellbeing workshop was delivered through Microsoft TEAMS. The facilitators shared their screen in order to show the psychoeducation resources, including a PowerPoint presentation and the sensory motor checklist. As it was not possible to do the practical exercise online, participants were encouraged to bring their own items for sensory toolkit. These items were shown to the participants and they spoke about their sensory benefits and how it affects their wellbeing. With participants who did not bring items, we were still able to discuss and describe the sensory items they found help them manage their sensory wellbeing. Afterwards, participants were sent the post-workshop evaluation questionnaire, the sensory wellbeing booklet and the sensory communication passport for them to complete in their own time. Details of the protocol and communication passports can be found on the following website: www.peacepathway.org.

\section{Data analysis}

Quantitative feedback from the patients was analysed with SPSS 27 using the Wilcoxon signed-rank test, which is recommended for small sample size and repeated measures [13]. A secondary analysis was then conducted to explore differences in outcomes between patients with and without high ASC traits using the Wilcoxon signed-rank test separately for the two groups. A significance level of 0.05 was selected. Effect sizes were reported in Cohen's $d$, with $d=0.2$, 0.5 and 0.8 corresponding to small, medium and large effects.

Qualitative data gained from the participants' responses to feedback questionnaires was analysed using inductive thematic analysis. Comments from both questions: 'what did you like most about this workshop?' and 'any other comments' were considered as a single dataset. Two researchers independently identified themes from the data before comparing and agreeing themes identified in a meeting.

\section{Results}

Six one-off sensory wellbeing workshops were delivered between February 2020 and February 2021. Three in the inpatient service, two in day care service and one open to patients in both treatment services. Two workshops were delivered online, the remainder in person. The in-person workshops were advertised to patients using posters and verbal invitations within treating services. The online workshops were promoted with flyers and an Eventbrite link shared with patients by email, and a Microsoft Teams link was emailed to registered participants one day before the workshop. The number of participants attending each workshop ranged from three to five, and there was no participant drop-out during sessions.

Of the twenty-seven patients who attended a sensory wellbeing workshop, twenty-three patients met criteria for inclusion in the study. Of the four patients excluded from the study, three were excluded as they did not have AN diagnosis (binge-eating disorder, bulimia nervosa), and one was excluded from analysis as they did not complete questionnaires at the end of the online workshop.

\section{Demographics}

Eighteen $(78 \%)$ had a diagnosis of AN restrictive subtype, and five (22\%) AN binge-purge subtype. The mean age of patient participants was 28.2 years $(\mathrm{SD}=9.6)$. The mean body mass index $(\mathrm{BMI})$ at admission to the service was $15.0(\mathrm{SD}=2.3)$. Eight patients (35\%) had received an AN diagnosis within the past 5 years, thirteen $(57 \%)$ more than 5 years ago, and 
Table 1 Change in sensory wellbeing measures after the workshop $(n=23)$

\begin{tabular}{|c|c|c|c|c|c|}
\hline Measures & $\begin{array}{l}\text { Mean score } \\
\text { at T1 } \\
\text { M (SD) }\end{array}$ & $\begin{array}{l}\text { Mean score } \\
\text { at T2 } \\
\mathrm{M}(\mathrm{SD})\end{array}$ & $Z$ & $p$ & $\begin{array}{l}\text { Co- } \\
\text { hen's } d\end{array}$ \\
\hline $\begin{array}{l}\text { Awareness of sen- } \\
\text { sory wellbeing }\end{array}$ & $3.00(1.24)$ & $4.09(0.79)$ & 3.354 & 0.001 & 0.97 \\
\hline $\begin{array}{l}\text { Awareness of the } \\
\text { strategies to en- } \\
\text { hance sensory well- } \\
\text { being }\end{array}$ & $2.70(1.15)$ & $4.04(0.71)$ & 3.684 & $<0.001$ & 1.21 \\
\hline $\begin{array}{l}\text { Confidence in man- } \\
\text { aging sensory well- } \\
\text { being }\end{array}$ & $2.48(0.95)$ & $3.78(0.85)$ & 3.800 & $<0.001$ & 1.41 \\
\hline
\end{tabular}

this data was not available for two patients. Seven (30\% of patients) scored highly $(\geq 6)$ on the AQ-10 at admission, indicating high autistic traits.

\section{Quantitative feedback from patients}

Twenty-three patients completed pre- (T1) and post (T2)-workshop questionnaires. There was strong evidence that self-reported awareness of sensory wellbeing, awareness of strategies to enhance sensory wellbeing, and confidence in managing sensory wellbeing increased after the workshops. The results are present in Table 1, with bar charts in Fig. 1.

Regarding "usefulness of the workshop", twentytwo (96\%) of patients rated it 3 ("Quite useful") to 5 ("Really useful").

\section{Differences in outcome between patients scoring high or low on the ASC measures}

Seven of the 23 participants scored highly $(\geq 6$ ) on the AQ-10 at admission, forming the high ASC group. The rest of the participants formed the low ASC group. There were no significant differences between the
Table 2 Outcome measures at T1 and T2 between patients scoring high and low on the ASC measure

\begin{tabular}{|c|c|c|c|c|c|c|c|}
\hline & \multicolumn{3}{|c|}{ T1 } & \multicolumn{4}{|l|}{ T2 } \\
\hline Measures & $n$ & Mean & SD & Mean & SD & $p$ & Cohen's d \\
\hline \multicolumn{8}{|c|}{ Low ASC group } \\
\hline Awareness & 16 & 3.00 & 1.21 & 4.00 & 0.82 & 0.004 & 1.04 \\
\hline Strategy & 16 & 2.50 & 1.16 & 4.06 & 0.77 & 0.001 & 1.62 \\
\hline Confidence & 16 & 2.38 & 1.03 & 3.75 & 0.93 & 0.001 & 1.55 \\
\hline \multicolumn{8}{|c|}{ High ASC group } \\
\hline Awareness & 7 & 3.00 & 1.41 & 4.29 & 0.76 & 0.066 & 0.86 \\
\hline Strategy & 7 & 3.14 & 1.07 & 4.00 & 0.58 & 0.131 & 0.64 \\
\hline Confidence & 7 & 2.71 & 0.76 & 3.86 & 0.69 & 0.039 & 1.07 \\
\hline
\end{tabular}

two groups in age, duration of illness, and on all T1 measures (awareness of sensory wellbeing, awareness of strategies to enhance sensory wellbeing, and confidence in managing sensory wellbeing). However, the high ASC group had higher BMI on admission (mean $=17.4, \mathrm{SD}=1.6$ ) compared to the low ASC group (mean $=13.9, \mathrm{SD}=1.8 ; p=0.001)$.

Table 2 displays the outcome measures between patients scoring high and low on the ASC measure. Wilcoxon signed-rank test revealed that there was significant improvement in all three measures in the low ASC group after the workshop (Awareness of sensory wellbeing: $p=0.004, d=1.04$; Awareness of strategies: $p=0.001, d=1.62$; Confidence in managing sensory wellbeing: $p=0.001, d=1.55$ ), all with large effect sizes. The high ASC group only showed significant improvement in confidence in managing sensory wellbeing $(p=0.039, d=1.07)$ and no significant change in the other two measures.
Fig. 1 Comparison of mean scores on the sensory wellbeing questionnaire measures at pre- (T1) and post-workshop (T2) $(n=23)$. Error bars $95 \%$ confidence interval. ${ }^{*}$ Level of significance based on Wilcoxon signed-rank tests: ** significant at $p<0.01$; *** significant at $p<0.001$

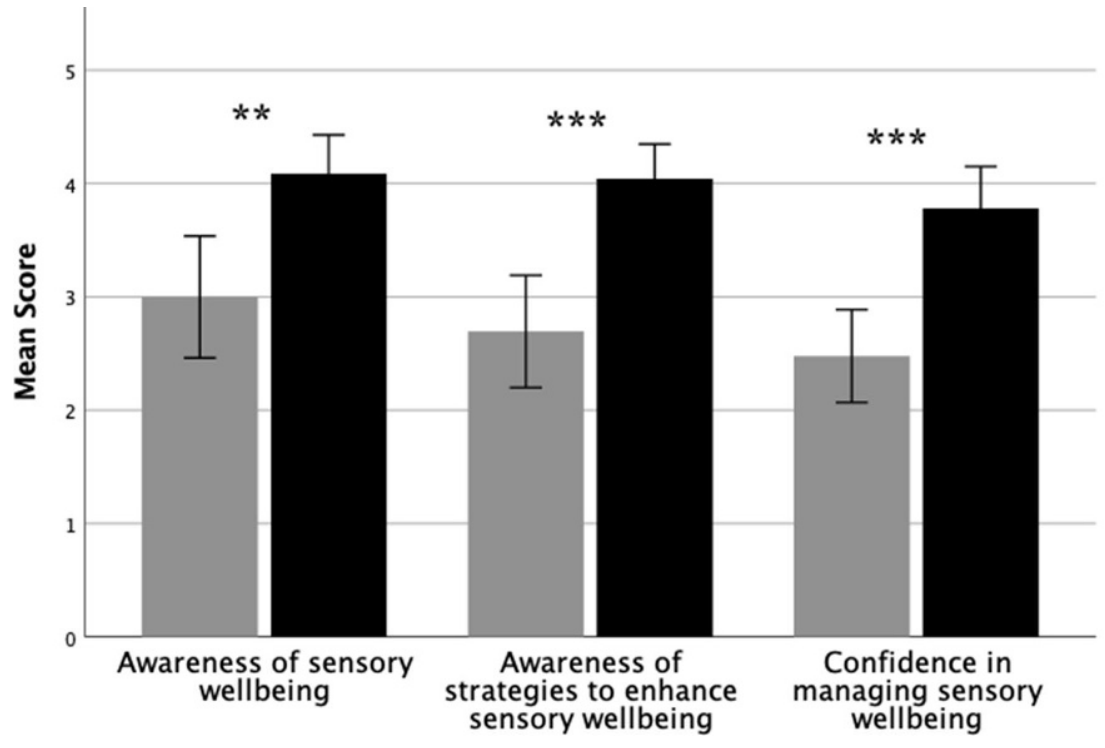


Table 3 A table of patient quotes providing examples for each theme

\begin{tabular}{|c|c|}
\hline Theme & Patient examples \\
\hline Enjoyable & $\begin{array}{l}\text { "It's the best thing I've attended since being on the ward" } \\
\text { "I enjoyed the opportunity to explore different areas of sensory } \\
\text { wellbeing which I would usually" } \\
\text { "This workshop lifted my mood significantly. I especially en- } \\
\text { joyed making the snowman, and also the smell of the cinna- } \\
\text { mon scented hand cream" "very informative and practical and } \\
\text { the sock snowman!" }\end{array}$ \\
\hline $\begin{array}{l}\text { Informa- } \\
\text { tive con- } \\
\text { tent and } \\
\text { activities }\end{array}$ & $\begin{array}{l}\text { "Being able to talk and discuss/connect with others and learn } \\
\text { about how others experience things-reminds me of how } \\
\text { unique we are and that unique part makes us who we are as } \\
\text { humans" } \\
\text { "Fun, informative, fascinating, useful" } \\
\text { "I realised how I do already use my senses to relax without } \\
\text { meaning to" } \\
\text { "Getting the chance to try out new toys etc. + practical work to } \\
\text { discover what I am particularly sensitive to" }\end{array}$ \\
\hline $\begin{array}{l}\text { Helpful } \\
\text { sensory } \\
\text { tools }\end{array}$ & $\begin{array}{l}\text { "Practical element of making own kit" } \\
\text { "It was lovely to have a few other soothing items to take } \\
\text { away, too, as well as the snowman and the hand-creams } \\
\text { (e.g. squishy man, tinsel, pompoms). Thank you so much for } \\
\text { organising this lovely session!" }\end{array}$ \\
\hline $\begin{array}{l}\text { Future im- } \\
\text { provement }\end{array}$ & $\begin{array}{l}\text { "Would love more scented oils for making hand creams" } \\
\text { "Could include sound/music and responses to that" } \\
\text { "Really needed more time. Would be good to follow up and } \\
\text { discuss findings fully" }\end{array}$ \\
\hline
\end{tabular}

\section{Qualitative feedback from the participants}

Qualitative feedback data was collected using openended questions in both inpatient and day-care programmes in order to improve future workshop content and delivery. Overall both written and verbal feedback was very positive, and facilitators observed that participants always wanted to stay beyond the scheduled finish time. Inductive thematic analysis was used to identify themes in the comments provided.

Four key themes were identified as summarised below (examples of quotes for each theme are highlighted in Table 3).

\section{Engaging}

All patients expressed that they enjoyed the sensory wellbeing workshop and reported that it was enjoyable and fascinating.

\section{Informative content and activities}

Patients reported that the psychoeducation materials shared and discussed in the workshop were very informative and helped give them an opportunity to explore different areas of sensory wellbeing.

\section{Helpful sensory tools}

Patients expressed that they found the DIY sensory tool box activities available during the workshop very helpful because they had real tools they could take away with them and use beyond the workshop to help with their sensory wellbeing (e.g. scented hand cream, squeeze toy).

\section{Future Improvements}

Patients suggested that future workshops include more sensory tools and activities that engage their senses and enhance their wellbeing. Many patients gave suggestions to use their own preferred sensory tools such as: scented oils and textile materials. Other comments suggest that patients would like the sensory wellbeing workshop to be run and available more regularly.

\section{Discussion}

There is increased research interest regarding the sensory systems and introspection of patients with EDs. Recent studies exploring ED and autism comorbidity have also created an interest in how ASC influences the sensory sensitivities in ED patients [4-6]. To our knowledge, research findings have not yet been translated into practical treatment tools [10]. In this paper, we have evaluated the practical use of sensory workshops for inpatient and day-care programmes and explored the response in patients with $\mathrm{AN}$ with and without ASC traits. The data from this study, along with previous research demonstrating positive patient experience and clinical outcomes in group therapies [14-16], indicated that there is feasibility for group workshops to be delivered as add-ons in the ED treatment programme.

The overall response to the workshop was positive, with participants reporting significant improvements in all measures post-workshop with large effect sizes. However, our results also highlight that in the group of patients scoring high on the ASC measure $(n=7)$, no significant change was reported in awareness of sensory wellbeing or awareness of the strategies to enhance sensory wellbeing after the workshop, although the medium to large effect sizes suggest that this is likely due to the power of the study. Nevertheless, this result is in line with previous research evidence that in a group therapy setting, patients with high ASC traits tend to show less preferable treatment response compared to patients with no ASC traits [17]. It is possible that the need for open, verbal discussions and the limited time for processing information in a group setting pose more difficulties for people with both AN and ASC traits, who often struggle with communication difficulties and social processing [11, 18]. Future workshops could be better adapted for this group of patients by allowing participants to write things down or type in chatboxes, and introducing more space in sessions to give participants more time to process information. Given the preliminary nature of the current study, future research on treatment response from patients with AN and ASC traits is warranted and will benefit from a larger sample size, improved workshop design and more controlled setting.

This pilot study also created ideas for improvement and development of future workshops including: increasing workshop duration, bringing more sensory tools and activities, and potentially introducing a follow up session, to provide space to reflect after completing the sensory booklet and sensory passport pro- 
vided and exploring sensory strategies outside of the workshop. Patient feedback highlighted the usefulness of regular sensory workshops, which might be made possible in future through collaborating across clinical services. As a one-off workshop this could also be provided to patients who are not otherwise involved in ongoing therapy services, such as those in severe and enduring eating disorders (SEED) physical monitoring programmes. Two of the in-person workshops were held during the current COVID-19 pandemic. This necessitated various adaptations, including ensuring that there was sufficient space for social distancing, use of hand sanitiser, limiting the use of shared materials, and thorough cleaning of any shared equipment between uses. For the online workshops that were held during the pandemic, adaptations included using online tools (SurveyMonkey, customised questions at registration on Eventbrite) for collecting outcome measures and an online booking system through Eventbrite. Compared to the in-person workshops, the online workshops were focused more around discussion, with patients sharing their own sensory experiences and strategies of enhancing their sensory wellbeing, due to the DIY element of the workshop being unfeasible. Despite this, holding the workshop online allowed for greater accessibility, including for patients receiving their treatment remotely, and therefore has the potential to reach more patients who may benefit from the workshop. Initially it was found that holding the workshop remotely highlighted some challenges, including creating awareness of the workshop, commitment to attending and ensuring patients were prepared with sensory items to discuss/share with the group. However, these issues were managed effectively by using the Eventbrite page to sign up and find information about the workshop and including more detailed examples of objects or strategies that may enhance sensory wellbeing to prompt discussion throughout the workshop. There is also the possibility of providing materials or a materials list ahead of time, so that patients attending the remote workshops are able to engage in a DIY activity. This was not trialled in the workshops discussed however presents a feasible adaptation for future workshops. Overall, all the COVID-19 adaptations were easily made, highlighting the flexibility of the workshop format.

Psychological interventions in a group format in general can bring unique benefits that are not achievable when working with patients individually. These benefits include sharing experiences and learning from others in a safe and therapeutic environment, being with other people and practicing interpersonal skills. Individuals with AN have difficulties making social contacts [19] and report high levels of social anhedonia-an absence of pleasure derived from being with people [20]. It has been observed that patients with AN often remain isolated and avoid communicating with other patients in inpatient settings.
Sensory workshop content is non-threatening and useful whilst also facilitating social communication.

The positive feedback elicited from patients on the feedback questionnaires highlights the wide acceptability of the group workshop. Patients generally found the group experience positive, and feedback from the workshop indicated that the majority of patients found it helpful. In particular, patients liked the interactive, easy nature of the workshop, as well as learning about different sensory experiences and how they have an impact on their lives. It is worth noting that the feedback form was not completed anonymously, which may have led to a positive response bias. That said, the positive feedback and acceptability of the intervention is promising, as poor treatment engagement is a common problem in existing psychological therapies [21].

This pilot study has some strengths worth mentioning: it is the first case series to report pilot work with sensory workshops, it contains a detailed protocol allowing others to replicate the workshop and that has allowed us to suggest improvements to the existing protocol, which paves the way for these workshops to be trialled in larger studies.

In terms of limitations, future studies would benefit from larger numbers of participants and more detailed information or measures used to capture change before and after the intervention. A follow-up session would also provide more insight into whether one standalone workshop provides lasting benefits for the patients. For specificity this study only included patients with an AN diagnosis and it would be valuable to investigate other ED diagnoses in future studies. It will be important for future studies to have clarity and analyse subgroups with and without ASC comorbidity to explore the question regarding similarities and differences in response to treatment. As described above, there is a risk of response bias in qualitative feedback as the patient responses were not anonymous.

Another limitation is the reduced number of participants in the online workshop, perhaps due to it being optional. As endorsed by one participant, a larger group may be useful to ensure a wider variety of individual experiences, thoughts and emotions to explore in relation to sensory wellbeing. Despite this, the results from the online workshop were similar to the inperson workshop. Participants reported an increase in their knowledge of their senses, and discussed ways of using their senses to manage their wellbeing. Another limitation to online delivery was the inability to explore new sensory items and create their own DIY sensory item and this was a hindrance to the overall experience of the workshop. However, the participants as well as staff were still able to bring some items that they already use and show these to the others, explaining how and why they are helpful for their sensory wellbeing. This also enabled further learning and consolidation of participants' awareness of senses 
and how items can be used to soothe or stimulate different senses.

\section{Conclusion}

Sensory wellbeing workshops seem to be a feasible format for patients with severe anorexia nervosa (AN). This pilot demonstrated that the workshop was able to enhance patients' awareness of their sensory wellbeing, strategies to enhance sensory wellbeing and their confidence in managing sensory wellbeing. Improving sensory awareness may help patients to manage distress, form healthy coping mechanisms supporting recovery from eating disorders (EDs), and live "sensationally" understanding their own sensory signature [22].

Acknowledgements Dr. K. Tchanturia would like to thank the Maudsley Charity for the assistance to conduct this study. We would like to thank staff and patients in the Eating Disorder Service in South London and Maudsley NHS foundation.

\section{Declarations}

Conflict of interest K. Tchanturia, C. Baillie, C. Biggs, A. Carr, A. Harrison, Z. Li, C. McFie, O. Oyeleye and C. Toloza declare that they have no competing interests.

Ethical standards The patient data had been collected as part of routine clinical practice on the Eating Disorders Unit (EDU); ethical approval was granted by an NHS Research Ethics Committee. Patients had been consulted and measures to be completed were reduced following their feedback about time and effort required.

\section{Appendix}

\section{Sensory wellbeing workshop protocol}

\section{Aims of the workshop}

- To raise awareness of the sensory system.

- To explore how the sensory system can help with self-regulation.

- To identify strategies that can enhance sensory wellbeing.

- To provide participants with the language and tools to communicate their sensory needs.

The workshop is designed to last between an hour and an hour and a half and should have at least two facilitators.

\section{Materials}

Flipchart paper, pens, small boxes or bags, pencils, play dough, stones, fidgety toys, textured fabrics, essential oils, hypoallergenic cream, small pots, ear plugs and any other sensory materials desired.

\section{Psychoeducation resource}

Our senses refer to the way in which we process and perceive the world around us in our brains. We all pro-
Table 4 Ideas for the sensory toolkit

Vision Sunglasses, tinted glasses

Sensory lights

Photos

Books/magazines

Optical illusions

Letters

Taking yourself somewhere visually appropriate for you (dark room/garden/greenspaces)

Sensory jars filled with glitter (Make your own: jar, glitter glue, glitter and water)

Touch Weighted lap pads/shoulder pads (Make your own: dried rice/ dried beans)

Fiddle toys: fidget spinner, fidget cubes

Textured items: scraps of fabric, (e.g. velvet, wool)

Spray bottle with water

Soft pillow or toy

Brush

Stress balls (Make your own: balloons filled with flour) BlueTak/play doh

Smell Scented oils (Make your own: get a plain body lotion and add essential oils)

Flowers

Potpourri (Dried plants e.g. lavender)

Perfume/room spray

Hearing Ear defenders, ear plugs. With items such as the ear defenders, it is important to moderate use and to use appropriately. It is possible if you over used ear defenders that when you don't use them, the more overly stimulating noise and noise sensitivity will be

Another option is audio isolating ear protectors. These essential 'focus' hearing for you to the conversation around

Headphones to play music that suits you and your level of arousal. It may be calming, soothing music or it may be heavy metal! You can also consider apps such as 'Headspace' and 'Calm' which have some free content. You can also listen to some white noise or other 'waves'

Make your own: voice recordings of family members/friends/ yourself saying things you know you like to hear when you are feeling this way. Filling a jar with dried rice/beans and creating a 'maraca'

Taste A mint spray (we acknowledge that it is most challenging area and for time being offer this strategy but open to more ideas and suggestions)

cess our senses slightly differently. Some people are hypersensitive, meaning they are highly sensitive, and some people are hyposensitive, meaning they have lowered sensitivity. You can experience hypersensitivity or hyposensitivity across different types of sensation: you might be hypersensitive to light, but hyposensitive to touch. (For more information please visit www.peacepathway.org).

Invite workshop members to discuss examples of their sensory sensitivity. Explore how different sensory sensations make them feel. Invite workshop members to discuss what makes their sensory sensitivities better or worse.

You can experience both heightened and lowered sensitivity to the same sensation depending on the 
1. How aware are you of your sensory wellbeing at the end of this workshop?

$\begin{array}{ccccc}\text { Not aware at all } & \text { Quite aware } & \text { Really aware } \\ 1 & 2 & 3 & 4 & 5\end{array}$

2. How aware are you of the strategies to enhance your sensory wellbeing as a result of the workshop?

$\begin{array}{ccccc}\text { Not aware at all } & \text { Quite aware } & \text { Really aware } \\ 1 & 2 & 3 & 4 & 5\end{array}$

3. How confident do you feel to manage your sensory wellbeing following the sensory wellbeing workshop?

$\begin{array}{ccccc}\text { Not confident at all } & \text { Quite confident } & \text { Really confident } \\ 1 & 2 & 3 & 4 & 5\end{array}$

4. How useful was this sensory workshop?

Not useful at all 1 2
Quite useful 3

Really useful

\section{5}

5. What did you like most about this sensory workshop?

6. Any other comments?

Fig. 2 Post-workshop sensory wellbeing questionnaire

context. Important factors which can inform our sensory responses include whether you are in control of the sensory stimuli, whether you are anxious or emotionally dysregulated, and whether there are lots of different stimuli at once in the environment.

Use previous examples from workshop members to stimulate discussion of how sensory sensations make them feel.

Our sensory sensations can make us feel better, or they can make us feel worse. This is closely related to self-regulation. Self-regulation is how we monitor and control our behaviour, emotions, and physiological arousal. Sensory sensations can impact our selfregulation. For example, if you are tired but need to focus at work you might turn up the lights (stimulating your visual system), or get up and walk around (stimulating your vestibular system). From the opposite perspective, if you're highly anxious and need to calm down you might retreat to a quiet space (soothing your auditory system), or rub a soft blanket (soothing your touch system).

Invite workshop members to fill out the SensoryMotor Preference Checklist (TherapyWorks, Inc.,
2018; https://www.alertprogram.com/wp-content/ uploads/2018/09/Sensory-Motor-Preference-Checkli st-revised-in-2018-PDF.pdf).

Workshop discussion: what sensations make us feel alert? What sensations make us feel calm?

This way, we can use sensations to change how we are feeling. However, we don't always have complete control over our sensory environments. If you get stuck in an environment with lots of negative sensory input this can be really overwhelming and unpleasant. If you can't avoid or escape that environment, you can use simple sensory strategies to help you feel calmer and more grounded. For example, taking a moment to smell an essential oil, or putting on headphones and listening to music to block out noise.

Invite discussion of different sensory strategies: what are small things you can do to change your sensory inputs?

Explain that aim of the workshop is to be aware, mindful of sensory system and live in harmony and make sensible adjustments when possible. 


\section{Sensory toolkit}

Practical exercise. Present different sensory materials (fabrics)/toys/tools/sensations to the group, and suggest to group members to create a sensory box with sensations that they find pleasurable. Include a DIY sensory exercise, for example creating a scented hand cream, select the fabric with the most pleasant texture, from variety of scents allow time to explore most soothing and enjoyable smell. Encourage group members to discuss the sensations throughout the DIY task. Whilst making sensory boxes (or bags), encourage group members to discuss what sensory tools they are choosing, and why.

If these kinds of tools do make a difference for you, it might be beneficial to let the people around you know about how you process sensory inputs and what strategies you use to help.

Hand out sensory communication passport worksheet.

Some sensory sensations that might help you selfregulate will not fit in a box. It might be helpful to think about and write down sensory strategies, such as going for a walk or going outside.

Hand out sensory booklet.

We all have different sensory thresholds. If we are aware of our sensory systems we are able to self-regulate by increasing or decreasing stimulation and making our environment work for us.

\section{Workshop resources}

Sensory toolkit: These are ideas you can develop yourself to help you manage your wellbeing. It may be that you are over-stimulated by certain senses or under-stimulated by certain senses. This can change from situation to situation and it might be a useful idea to include items which will increase and decrease your arousal levels (Table 4).

\section{References}

1. Tchanturia K, Hambrook D, Curtis H, Jones T, Lounes N, Fenn K, et al. Work and social adjustment in patients with anorexia nervosa. Compr Psychiatry. 2013;54(1):41-5.

2. Arcelus J, Mitchell AJ, Wales J, Nielsen S. Mortality rates in patients with anorexia nervosa and other eating disorders: a meta-analysis of 36 studies. Arch Gen Psychiatry. 2011;68(7):724-31.

3. Brockmeyer T, Friederich H, Schmidt U. Advances in the treatment of anorexia nervosa: a review of established and emerging interventions. Psychol Med. 2018;48(8):1228.

4. Kinnaird E, Dandil Y, Li Z, Smith K, Pimblett C, Agbalaya R, et al. Pragmatic sensory screening in anorexia nervosa and associations with autistic traits. J Clin Med. 2020;9(4):1182.

5. Kinnaird E, Stewart C, Tchanturia K. Taste sensitivity in anorexia nervosa: a systematic review. Int J Eat Disord. 2018;51(8):771-84.
6. Kinnaird E, Tchanturia K. Looking beneath the surface: Distinguishing between common features in autism and anorexia nervosa. JBehav Cogn Ther. 2021;31(1):3-13.

7. Li Z, Dandil Y, Toloza C, Carr A, Oyeleye O, Kinnaird E, et al. Measuring clinical efficacy through the lens of audit data in different adult eating disorder treatment programmes. FrontPsychiatry. 2020;11:599945. https://doi.org/10.3389/ fpsyt.2020.599945.

8. Kinnaird E. What's that smell? 2020. https://www. peacepathway.org/blog/whats-that-smell. Accessed 29 Jan 2021.

9. Tchanturia K, Smith C, Glennon D, Burhouse A. Towards an improved understanding of the anorexia nervosa and autism spectrum comorbidity: PEACE pathway implementation. Front Psychiatry. 2020; https://doi.org/10.3389/ fpsyt.2020.00640.

10. Kinnaird E, McLachnan I, Smith K, Tchanturia K. Assessing sensory sensitivities co-occurring in autism and eating disorders. London: Jessica Kingsley; 2021.

11. APA. Diagnostic and statistical manual of mental disorders (DSM-5 $\left.{ }^{\circledR}\right)$. : American Psychiatric; 2013.

12. Allison C, Auyeung B, Baron-Cohen S. Toward brief "red flags" for autism screening: the short autism spectrum quotient and the short quantitative checklist for autism in toddlers in 1,000 cases and 3,000 controls [corrected. J Am Acad Child Adolesc Psychiatry. 2012;51(2):202-212.e7.

13. Field A. Discovering statistics using IBM SPSS statistics. Introducing statistical methods series. London: SAGE; 2009.

14. Sparrow KA, Tchanturia K. Inpatient brief group therapy for anorexia nervosa: patient experience. Int J Group Psychother. 2016;66(3):431-42.

15. Tchanturia K, Larsson E, Brown A. Benefits of group cognitive remediation therapy in anorexia nervosa: case series. Neuropsychiatr. 2016;30(1):42-9.

16. Ohmann S, Popow C, Wurzer M, Karwautz A, Sackl-Pammer P, Schuch B. Emotional aspects of anorexia nervosa: results of prospective naturalistic cognitive behavioral group therapy. Neuropsychiatr. 2013;27(3):119-28.

17. Tchanturia K, Larsson E, Adamson J. How anorexia nervosa patients with high and low autistic traits respond to group cognitive remediation therapy. BMC Psychiatry. 2016;16:334. https://doi.org/10.1186/s12888-016-1044-x.

18. Kinnaird E, Norton C, Stewart C, Tchanturia K. Same behaviours, different reasons: what do patients with cooccurring anorexia and autism want from treatment? Int Rev Psychiatry. 2019;31(4):308-17.

19. Cardi V, Mallorqui-Bague N, Albano G, Monteleone AM, Fernandez-Aranda F, Treasure J. Social difficulties as risk and maintaining factors in anorexia nervosa: a mixedmethod investigation. Front Psychiatry. 2018;9:12.

20. Harrison A, Mountford VA, Tchanturia K. Social anhedonia and work and social functioning in the acute and recovered phases of eating disorders. Psychiatry Res. 2014;218(12):187-94

21. Hay P. Current approach to eating disorders: a clinical update. Intern Med J. 2020;50:24-9.

22. Dunn W. Living sensationally: understanding your senses. London: Jessica Kingsley; 2007.

Publisher's Note Springer Nature remains neutral with regard to jurisdictional claims in published maps and institutional affiliations. 